\title{
Compromiso del profesorado universitario con estudiantes de Grado de Educación Primaria en tiempos de pandemia: Un estudio de caso
}

\section{University faculty engagement with undergraduate Primary Education students in pandemic conditions: A case study}

\author{
Rocío Quijano López ${ }^{1}$, Miguel Pérez Ferra², Ma del Carmen Martínez Serrano ${ }^{3}$ \\ ${ }^{1}$ Universidad de Jaén. rquijano@ujaen.es \\ ${ }^{2}$ Universidad de Jaén.mperez@ujaen.es \\ ${ }^{3}$ Universidad de Jaén.mcmartin@ujaen.es
}

Recibido: $21 / 6 / 2021$

Aceptado: 22/10/2021

Copyright (C)

Facultad de CC. de la Educación y Deporte. Universidad de Vigo

\section{(i) $\$$}

Dirección de contacto:

Rocío Quijano López

Facultad de Humanidades y Ciencias de la

Educación (Edif. D2-341)

Universidad de Jaén

Campus Las Lagunillas, s/n

23071 Jaén

\section{Resumen}

El estudio analiza treinta y una narrativas de estudiantes del título de grado de Maestro en Educación Primaria de la Universidad de Jaén, que describen la percepción de los estudiantes sobre el nuevo contexto formativo ante el COVID-19, los problemas generados y la respuesta de profesores y alumnos ante la nueva situación. Se parte de treinta y una narrativas autobiográficas de los estudiantes que siguen el curso en línea, utilizando un proceso de análisis inductivo, basado en la "Teoría Fundamentada", fundamentado en un estudio descriptivo mediante el software informático NVivo-11Plus, que ha generado seis categorías relacionadas con la adecuación del profesorado para formar en línea, compromiso ético de profesores y estudiantes, interacción, así como nuevos contextos y problemas que surgen. El discurso de los estudiantes valora positivamente el esfuerzo de actualización de los docentes, su compromiso ético y capacidad para la interacción. El discurso no evidencia compromiso ético de los estudiantes, aunque sí cierta pulsión emocional hacia el "otro". Se evidencian carencias al considerar la actividad formativa como mera efectuación y fragmentación a partir de la filosofía analítica del lenguaje, carencias que se manifiestan en la integridad e integralidad de la formación de las personas.

\section{Palabras clave}

Formación Virtual de Estudiantes, Actualización Profesional Docente, Compromiso Ético, Interacción en los Procesos Formativos 
The study analyses thirty-one students' narratives from the Primary Education degree at the University of Jaén, which describe their perception of the new formative context faced with the COVID-19, the problems generated and the response of teachers and students to the new context. Thirty-one autobiographical narratives of students who follow the online course are used as the basis for the study. An inductive analysis process, based on "grounded theory", was used, based on a descriptive study using NVivo-11Plus software, which generated six categories related to the suitability of the teaching staff for online training, ethical commitment of teachers and students, the interaction, as well as the new contexts and problems generated. The students' discourse values positively the effort of updating teachers, their ethical commitment and capacity for interaction. Their discourses do not show students' ethical commitment, although there is a certain emotional impulse towards the "other". Their discourse show shortcomings when they consider the formative activity as mere efficacy and fragmentation based on the analytical philosophy of language, shortcomings that are manifested in the integrity and integrality of the students training.

\section{Key Words}

Distance Education, Inservice Teacher Education, Ethical Instruction, Instructional Effectiveness

\section{INTRODUCCIÓN}

Mediante Resolución Rectoral de 9 de noviembre de 2020, se suspendían las clases presenciales en los títulos de grado de la Universidad de Jaén, a fin de prevenir los contagios por COVID 19. Las medidas de confinamiento han definido un nuevo modo de llevar a cabo el ejercicio profesional docente y la formación de los estudiantes, que ha demandado del profesorado una preparación adicional para desarrollar la actividad formativa en línea en nuevos contextos de aprendizaje y para la reflexión sobre problemas adicionales y distintos, generados por la pandemia del COVID-19.

La situación descrita ha demandado un ejercicio adicional de la ética profesional docente, no solo para conocer nuevos recursos y materiales telemáticos o desarrollar la docencia de modo más adecuado a los recursos disponibles y a las nuevas situaciones de aprendizaje, y para facilitar la interacción entre estudiantes y entre estudiantes y profesores en nuevos contextos de aprendizaje (Brito-Lara y Herrera-Rendón, 2021), sino también para estar atento al deterioro psicosomático que está generando esta situación, a la calidad de los dispositivos o a la señal para acceder a la docencia digital

Los considerandos descritos no solo se han planteado con la urgencia que demandaba la situación sobrevenida mediante el COVID-19, sino también por una gestión desacertada de las universidades, en la medida que han interpretado el "salto" tecnológico como mera incorporación de dispositivos o herramientas digitales, obviando la superación de la dicotomía dualismo digital-no digital (Coeckelbergh, 2020).

El motivo aducido conduce a conocer la voz de los estudiantes sobre cómo han percibido la disposición y compromiso del profesorado y de ellos mismos ante estos cambios y cómo han reaccionado ante los mismos, sobre todo, cuando el tema que nos ocupa supera la mera formación digital actualizada de los docentes y aborda la carencia institucional de una visión holística del problema de la formación en estas circunstancias, cuya consecuencia es que el profesorado no cuenta con el apoyo 
sustantivo y clarificador que le permita asumir de modo diáfano la propuesta formativa de sus alumnos (Fernández-Regueira et al., 2020).

\section{MARCO TEÓRICO Y REVISIÓN DE LA BIBLIOGRAFÍA}

La situación descrita lleva a realizar una revisión de la bibliografía científica sobre el tema objeto de estudio, que permita aportar referencias justificativas y contrastar los resultados obtenidos en esta investigación con otras similares.

Se definieron aspectos como el comportamiento ante la incorporación de recursos y herramientas tecnológicas por profesores y estudiantes en una situación no solicitada, sino urgida; las reflexiones de los estudiantes sobre el compromiso ético de los docentes para abordar esta nueva andadura; la posibilidad de los estudiantes de interaccionar de otro modo con compañeros y profesores, para corregir las carencias que supone la supresión de la proximidad física y el compromiso ético de los estudiantes ante este nuevo contexto de desarrollo de la enseñanza, así como la descripción del nuevo formato de su actividad en este nuevo contexto y cómo piensan que se han abordado los problemas que se han generado en el mismo.

Los estudiantes constatan en su discurso el esfuerzo que las universidades han realizado para dotar a aquellos profesores y alumnos que lo han demandado de dispositivos digitales, e incluso de resortes que les han permitido disponer de una señal adecuada. El alumnado valora, en líneas generales y positivamente, el esfuerzo que los docentes han realizado para el dominio de nuevos dispositivos. Sin embargo, plantean carencias en la utilización pedagógico-tecnológica de los dispositivos (Cabero y Llorente-Cejudo, 2020; Romero, et al., 2019), que se acentúa en acciones formativas a distancia, para las que el docente debe disponer de competencias específicas, tales como: capacitación para indagar sobre los temas a trabajar, saber estructurar los contenidos que demandan conocimiento profundo sobre la realidad ontológica de la materia; capacitación para diseñar tareas individualizadas y en grupo para el aprendizaje, conocimiento didáctico y dominio de la comunicación asincrónica y sincrónica o saber diseñar una clase en línea, entre otros aspectos (Marciniak, 2015).

Conviene indicar que algunos de los déficit indicados por los estudiantes tienen una causa próxima y otra remota; la primera, determinada por la urgencia de una situación sobrevenida que no ha permitido una formación adecuada en un tiempo breve ni con la profundidad necesaria sobre las competencias que se deben adquirir, generando -en no pocas ocasiones- prevenciones, incertidumbres y agotamiento ante el nuevo sistema de formación (Suárez-Monzón, 2020); la segunda causa, ya referida en la introducción, está determinada por la consideración de las universidades de que el "salto" tecnológico se aborda exclusivamente con la dotación de dispositivos digitales y software informático (Coeckelbergh, 2020).

Estudios como el de Álvarez-Flores y Núñez (2019) ponen de manifiesto las dificultades de los estudiantes ante el trabajo en línea en aspectos como el seguimiento de las clases o realización de actividades de carácter colaborativo. Otros estudios constatan que la causa de estos problemas está en el error de considerar a los estudiantes como nativos digitales de modo apriorístico, por el solo hecho de pertenecer a una generación determinada, cuando en realidad carecen de competencias para la utilización 
de las tecnologías aplicadas al ámbito de la formación, ya que como está evidenciando el tiempo de pandemia y las investigaciones "ad hoc", los estudiantes utilizan un número limitado de dispositivos que destinan a actividades de ocio y no a acciones formativas (Belçaguy et al., 2020; Bossolasco et al., 2020). En un estudio reciente, relativo a un proyecto de $\mathrm{I}+\mathrm{D}+\mathrm{i}$, Pérez-Ferra et al., (2019), se ponían de manifiesto las carencias de los estudiantes que inician los estudios de Maestro respecto a la competencia digital, así como a la competencia comunicativa y la correlación que existe en el desarrollo simultáneo de ambas.

Lo descrito en las narrativas de los informantes demanda la necesaria previsión en la formación del alumnado en las competencias digitales y en la prevención de adicciones y mal uso de las tecnologías (García-Areitio, 2021), considerandos en los que ha incidido la Junta de Andalucía (Consejería de Educación, Cultura y Deporte, 2015) en la Orden de 17 de marzo de 2015, que regula el currículum de Educación Primaria, indicando que se ha de incidir en “ (...) la previsión de formación del alumnado en las necesarias competencias digitales y en la prevención de adicciones y mal uso de las tecnologías" (p. 764). Sin embargo, las carencias en la formación específica del profesorado, no han permitido la actualización de las intenciones que refleja esta orden a ese respecto.

Aunque la revisión manifiesta que las vivencias de los estudiantes se concretan de modo más notorio en la impartición de clases, las capacidades didácticas de los docentes y en las aptitudes que tienen para la utilización de los dispositivos digitales, es relevante la consideración de otros aspectos que reflejan en sus narrativas, en relación a: cómo contribuye a discernir la importancia y el origen del compromiso ético del profesorado; la pérdida de la comunicación y la interacción por la ausencia de proximidad física; o la corresponsabilidad de los estudiantes ante los problemas que genera esta situación.

También hay estudios realizados en Estados Unidos y China, donde se refleja aunque en una minoría de estudiantes, una visión más humanística, que transciende a lo meramente académico. Un estudio de Cao et al., (2020) aborda las variables que más afectan a la calidad de la enseñanza, tales como emociones negativas o la incertidumbre ante un posible contagio, que generan estrés o desasosiego. En otro estudio llevado a cabo por Garris y Fleck (2020) los estudiantes valoran negativamente el semestre, calificando los cursos menos interesantes, pero sobre todo por el deterioro psicológico que había generado el confinamiento en muchos de ellos.

La realidad descrita pone de manifiesto que hay estudiantes que van más allá en la consideración de la función de la escuela como mera dinamizadora de un “(...) conjunto de tareas y rutinas que se llevan a cabo de acuerdo a un diseño curricular y unas estrategias metodológicas" (Rivas, 2014, p. 490). Suelen ser estudiantes que realizan actividades de voluntariado basadas en la acogida al "otro".

Otras investigaciones destacan la formación en tiempo record de gran parte del profesorado, partiendo de un compromiso moral que transciende la racionalidad instrumental (Buxarrais y Carreño, 2019; Martínez-Martín y Carreño Rojas, 2020). Este reconocimiento por los alumnos, denota una formación de su identidad basada en el respeto y la consideración al "otro", adquirida en sus vivencias, día a día, en la familia y en el ejemplo de quienes les formaron, aspectos esenciales para el desarrollo de su vocación, entendida como "el compromiso con los 'otros'” (Day, 2006, p. 22). 
Los estudiantes valoran positivamente el esfuerzo realizado por gran parte de los docentes para mantener un clima de comunicación e interacción favorables con los estudiantes y entre los estudiantes. Refieren en la mayoría de las ocasiones una comunicación previa a la interacción, que pasa por un nivel de excelencia docente. Aluden a un profesorado con conocimiento de la materia, que se ocupa de conocer si han entendido lo explicado, que no evidencia monotonía en su discurso para enseñar, y que fundamenta lo explicado. Consideran que ese clima favorable en la comunicación durante la enseñanza es decisivo para generar un ambiente favorable, de confianza e interacción entre docente y estudiantes y, transversalmente, entre estudiantes (Alonso, 2019; Monroy-Farías, 2020).

Otras investigaciones inciden en que la percepción de los estudiantes para generar procesos de interacción se fundamenta en la preparación previa de los docentes, indicando que la generación de comunicación y posterior interacción entre profesores y estudiantes y entre estudiantes tiene como precedentes una enseñanza de calidad, en la que se ha de generar una comunicación fluida, evidencia de responsabilidad, preparación previa de las clases, dominio de la materia y de métodos para que los estudiantes puedan interesarse y comprender los contenidos, desarrollar capacidades y adquirir competencias, así como actividades que favorezcan el desarrollo de competencias específicas y transversales (Calvo y Susinos, 2010; Martín, 2019).

Respecto al compromiso ético de los estudiantes, hay estudios que reflejan su colaboración con el profesorado para que las clases sean más dinámicas e interactivas (De Dios, 2018). En otros se pone de manifiesto que el alumnado ha contribuido al desarrollo del compromiso y la responsabilidad ante el grupo, y ha reflexionado sobre las implicaciones de su conducta en la realidad social del grupo, para favorecer la convivencia y solidaridad (De la Calle-Maldonado et al., 2021; Garrote-Rojas et al., 2019), aspectos no impulsados por una compasión efímera, sino por creencias de corresponsabilidad (García-Sanz et al., 2010; Tejedor et al., 2020). Sin embargo, habría que ir más allá e indicar que este sentido de compromiso moral de los docentes tiene su fundamento en las reflexiones plenamente actuales de Ortega-Ruiz (2004):

(...) la relación más radical y originaria que se establece entre maestro y alumno, en una situación educativa, es una relación ética que se traduce en una actitud de acogida y un compromiso con el educando, es decir, hacerse cargo de él. En el núcleo mismo de la acción educativa no está, por tanto, la relación profesional-técnica del experto en la enseñanza, sino la relación ética que la define y constituye como tal acción educativa (p. 5).

Las reflexiones del profesor Ortega llevan a considerar que los estudiantes, aunque tácitamente, valoran un nuevo modo de enfocar la educación, lo que supone definir los procesos formativos no desde la consideración del sujeto autónomo, propio del "idealismo kantiano", sino desde el sentido de alteridad y acogida del otro, "que nos constituye en sujetos morales cuando respondemos de él” (Ortega-Ruiz, p. 5). Y es aquí donde se halla la centralidad de la relación profesor-alumno y alumno-alumno, en el sentido de acogida y corresponsabilidad.

\section{RELATO METODOLÓGICO}

\subsection{Objetivos de la investigación}


Los objetivos de la investigación se orientaron a conocer la percepción de los estudiantes sobre el nuevo contexto formativo, los problemas que genera y las respuestas que aportan profesores y estudiantes a la nueva situación. Atendiendo a lo cual, quedan concretados en:

- Determinar y analizar la estructura del discurso de los estudiantes respecto a su formación en tiempos de pandemia.

- Reconocer las claves filosóficas e indicadores culturales que sustentan el discurso de los estudiantes respecto al compromiso del profesorado con el alumnado.

\subsection{Proceso metodológico}

El estudio se inicia mediante el análisis de narrativas autobiográficas de estudiantes del primer curso del Título de Grado de Maestro en Educación Primaria de la Universidad de Jaén, respecto a su percepción de la situación académica ante el nuevo estado de pandemia; las creencias que definen esa percepción y las corrientes de pensamiento que las determinan, aspectos que facilitan una perspectiva reflexiva en los estudiantes, definitoria de la finalidad de su discurso (Arias-Sanabria et al., 2016). A este respecto es interesante considerar que un gran porcentaje de estos estudiantes son la primera generación universitaria de sus familias.

Previamente a la confección de las narrativas se llevó a cabo un taller en el que se les indicó a los estudiantes que deberían reflejar su percepción sobre el desarrollo de la acción docente en la realidad actual, describiendo sus vivencias. Simultáneamente se les explicó la finalidad de la investigación, siendo voluntaria la realización de la narrativa y no figurando ningún elemento identificativo personal de los estudiantes en la misma. Las narrativas se entregaron al concluir el mes de febrero de 2021.

Se ha llevado a cabo un estudio cualitativo, en cuanto que se busca dar respuesta a procesos singulares desde la unicidad e individualidad de cada informante (Shakouri, 2014).

La información facilitada por las narrativas autobiográficas de los estudiantes se ha analizado mediante la "Teoría Fundamentada", que ha facilitado la definición de categorías a través de procedimientos inductivos, cuya finalidad es: "La apuesta por la construcción de teorías inductivas a partir de los discursos de actores involucrados" (Alarcón-Lora et al., 2017). Sin embargo, la actualidad en la investigación cualitativa demanda mayor precisión, analizándose las narrativas desde la vertiente de la "Teoría Sustantiva" de la "Teoría Fundamentada", que, sin desvincularla de su posicionamiento inicial, considera además la realidad cultural de un área sustantiva (Apramian et al., 2017), en este caso la educativa y desde la realidad cultural del Humanismo Cívico.

\subsection{Definición de los casos objeto de estudio y diseño de la información}

\subsubsection{Definición de los casos de objeto de estudio}

La población ha quedado integrada por 164 estudiantes del título de Grado de Educación Primaria, participando en el taller inicial 78 y seleccionando 31 de las narrativas aportadas, atendiendo a la calidad de las mismas. 


\subsubsection{Diseño y análisis de la información}

Se ha llevado a cabo un diseño descriptivo, definido por una marca de nube para determinar la estructura y significación semántica de los elementos del discurso; en segundo lugar, un conteo de palabras, que determina su frecuencia en el discurso (Strauss y Corbin, 2016); y por último, se realizó una matriz de codificación sobre frecuencias cruzadas entre los nodos resultantes de proceso de análisis inductivo de las categorías resultantes, relativos a la concepción que tienen los estudiantes sobre el nuevo contexto de formación en línea, procedimiento que facilita el conocimiento e incidencia que tienen unas categorías en la estructura de otras (Herrera-Pastor, 2016), y sus correlaciones e implicaciones en el discurso de los estudiantes.

El análisis de la información se realizó mediante el software informático NVivo11Plus. En la primera fase se procedió a la estructuración del discurso mediante la definición de las categorías más significativas, a fin de determinar la idea central del discurso, los procesos de subordinación, considerando las temáticas implícitas y relación semántica entre las mismas.

En una segunda fase se ha analizado el uso de las palabras que articulan las narrativas, la estructura proposicional de las mismas y su incidencia en la coherencia del discurso, así como las posibles inferencias lógicas en las narraciones, fundamentales para la comprensión del discurso, en la medida que determinan la información no explicitada en el texto (González-García, 2009), ayudando a definir la coherencia del discurso.

Posteriormente, y mediante el "método de comparación constante" se procedió a generar teoría a partir del análisis comparativo y sistémico de los datos, que condujo a la identificación y relación de conceptos para interpretar el discurso de los estudiantes (Hernández-Flores y Carrera-García, 2019) e incluso permitió, a partir de generalizaciones fundamentadas sobre un grupo particular, generar teoría en niveles más altos de abstracción (Pawluch, 2005).

\subsection{Caracterización del discurso de los estudiantes}

El discurso de los estudiantes ha quedado determinado por 6 categorías, que definen las vivencias de los estudiantes ante el desarrollo de la actividad académica en tiempos de pandemia. Hay predominio de la categoría "Compromiso Ético del Profesorado" (CEP), que se materializa de modo más acentuado, con la "Formación Docente para la Formación en Línea" (FPDL) y con la "Interacción Profesor-Alumno" (IPA), como los dos aspectos clave en los que ha de incidir el compromiso ético docente.

Siguen argumentando los estudiantes, que el compromiso ético se vincula fundamentalmente a la actividad desarrollada en clase por el docente y está determinado por las acciones de mejora emprendidas por el profesorado, como facilitador de los procesos instructivos, orientados al aprendizaje de los alumnos, situación que queda reflejada en la "marca de nube", Figura 1, y en la Tabla 2, que recoge el porcentaje de sustantivos más utilizados por los estudiantes en sus narrativas. En menor medida consideran de vital importancia la "interacción profesor-alumno". Sin embargo, la ponderan en lo meramente instructivo, obviando aspectos emocionales, organizativos e instrumentales (Pianta y Hamre, 2009). 
La teoría surgida de las relaciones entre las categorías definidas inductivamente se vincula con la revisión de tareas o la atención individualizada de los estudiantes (Fernández-García et al., 2021; Gares et al., 2020). Pero manifiesta la omisión en el discurso de los estudiantes de situaciones y aspectos externos a la relación académica entre profesores y estudiantes, que sí inciden significativamente en el desenvolvimiento académico. Aspectos como: adicciones, dificultades económicas, situaciones de depresión o los obstáculos que les genera un trabajo paralelo a los estudios, etc., obviando una categoría tan representativa como es "Problemas que Genera la Nueva Situación", (PGNS) de muy baja incidencia en las narrativas de los estudiantes (Aristovnik, et al. 2020).

Las inferencias teóricas permiten afirmar que el perfil del discurso de los estudiantes que acceden a la Universidad desde los estudios de Secundaria se vincula más al estudiante como centro del proceso de aprendizaje, que como centro de formación, lo que denota que no conocen la realidad última del vínculo entre estudiantes y profesores, que nos define como sujetos morales cuando respondemos del otro (Figura 1).

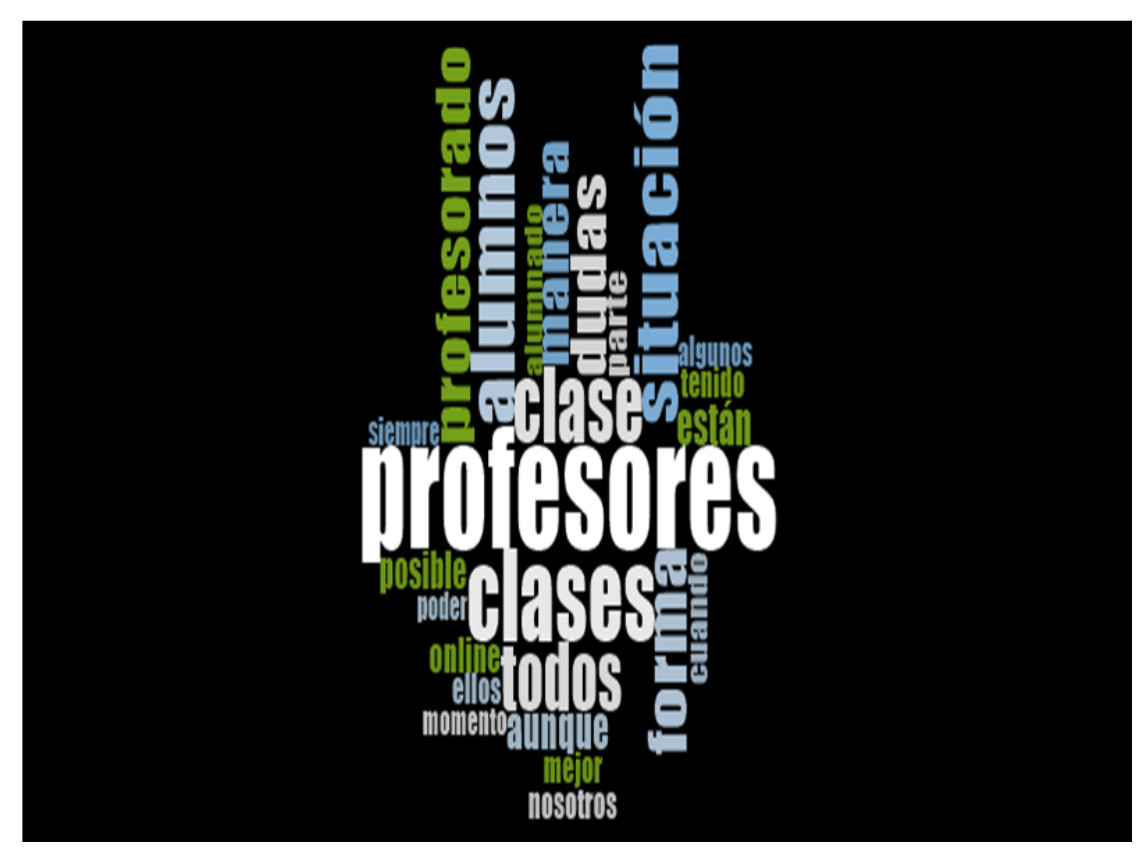

Figura 1. Marca de nube que determinar la estructura y significación semántica de los elementos del discurso de los estudiantes sobre nuevos contextos de aprendizaje ante el COVID-19

\begin{tabular}{lccl}
\hline \multicolumn{1}{c}{ Palabra } & Conteo & Porcentaje ponderado (\%) & \multicolumn{1}{c}{ Palabras similares } \\
\hline Profesores & 227 & 2,09 & Profesorado, ellos, están, todos \\
\hline Clase & 150 & 1,40 & Clases \\
\hline Alumnos & 93 & 0,97 & Alumnado \\
\hline Situación & 67 & 0,63 & Contexto \\
\hline Forma & 58 & 0,54 & Modo \\
\hline Dudas & 54 & 0,50 & Indecisión \\
\hline Disponibilidad & 151 & 1,41 & Apoyo, dedicación \\
\hline
\end{tabular}

Tabla 1. Sustantivos con mayor frecuencia en el discurso de los estudiantes respecto a los nuevos contextos de aprendizaje ante el COVID-19 
3.5. Estructura del discurso de los estudiantes en torno a sus vivencias sobre el nuevo contexto docente ante el estado de pandemia

Los nodos definidos mediante un proceso inductivo, atendiendo a la naturaleza semántica de las narrativas autobiográficas, han generado una realidad teórica definida por las frecuencias recogidas en la Tabla 2 y las interacciones del gráfico de redes de la Figura 2. A partir de tales criterios, se analizó el discurso y las interacciones entre categorías que definen la estructura del discurso de los estudiantes.

\subsubsection{Discurso de los estudiantes sobre la adecuación para la formación en línea}

Se considera que el profesorado está haciendo un esfuerzo para adaptarse a la nueva situación, aunque no es menos cierto que lo están haciendo en un tiempo record y con una inflación de información como aspectos que hay que tener en cuenta en contra de ese proceso (Inf. 4; 12; 13):

“(...) considero que el profesorado se está adaptado a esta nueva situación lo mejor que puede, haciendo todo lo que está en su mano. Sí es verdad que a muchos de los profesores les está costando un poco más todo esto de tener que hacer las clases en línea” (Inf. 4).

En líneas generales, los estudiantes se encuentran cómodos pues los profesores responden a sus dudas (Inf. 4;3;13). Sin embargo también hay sus excepciones, aunque no frecuentes (Inf. 5; 9; 13):

"Me encuentro cómoda con la mayoría de profesores, aunque hay excepciones, y que no me resuelven dudas o incluso no incluyen a los alumnos en sus clases, lo cual me parece una falta de respeto y una dejadez por su profesión" (Inf. 5).

Aunque, en líneas generales, los estudiantes muestran plena satisfacción con el esfuerzo que hace el profesorado por impartir los contenidos de modo adecuado, generar interacción y atenderles individualmente, si bien es cierto que definen diferentes niveles de compromiso docente (Inf. 2; 6, 9; 18;22;31):

"En referencia a las clases, hay algunos profesores que las tienen mejor preparadas, ya que hace el temario más didáctico, mediante encuestas, kahoot o algún tipo de juego y todo ello te motiva mucho más” (Inf. 31).

Pero, en líneas generales, se describen aspectos que denotan un gran esfuerzo por adaptarse didácticamente a la nueva situación docente, utilizando foros, presentaciones, etc. (Inf. $17 ; 25 ; 28 ; 31$ ):

"Creo que se han adaptado en un tiempo record a todo lo que se les ha venido encima y están haciendo una gran labor con las herramientas proporcionadas, lo que pone de manifiesto su dedicación y compromiso hacia nosotros” (Inf. 30).

Valoran el sentido vocacional que hay detrás del esfuerzo docente, vinculado en algunos casos con ser maestro. Conocen qué profesores y profesoras son maestros y ven en el "motor" de su esfuerzo el compromiso con sus alumnos y la gratificación que les reporta tratarles adecuadamente (Day, 2006) (Inf. 13; 14; 15; 21):

"Hay algún profesor que se siente contento cuando entendemos lo que nos aclara. Y se implica todavía más cuando ve que tenemos una dificultad, incluso a nivel personal" (Inf. 21). 


\subsubsection{Discurso de los estudiantes sobre el compromiso ético del profesorado}

El discurso de los estudiantes vincula el compromiso ético de los docentes de modo más acentuado con la adecuación para la formación docente en línea y, en un segundo lugar con la interacción entre profesores y alumnos.

Los estudiantes, en una gran mayoría, indican en su discurso que el profesorado se manifiesta próximo en las videoconferencias (Inf. $13 ; 15 ; 28 ; 31$ ), y se preocupa de su situación personal (Inf. 15, 28; 29; 30; 31):

"No se ha perdido la proximidad de los profesores en el trato con los alumnos, son amables y se preocupan por nuestra salud, además de dar consejos para nuestra seguridad. Es admirable la rapidez con la que responden a las dudas” (Inf. 28).

Pero el compromiso no es similar en todos (Inf. 13; 7;8; 17), algunos de ellos remiten a los materiales de la plataforma:

"No todos los profesores te resuelven las dudas que te surgen, simplemente se basan en indicarte que todo el material está en docencia virtual o en secretaria” (Inf. 17).

Consideran los estudiantes que el profesorado ha intentado ser más próximo e interaccionar con los estudiantes (Info. 18):

"El profesorado creo que bajo la experiencia que yo vivo está dando todo de su parte. Los noto más familiares y cercanos, preocupándose de temas académicos y personales” (Inf. 18).

Pero no es menos cierto que hay otros que, aunque manifiestan buenas intenciones, no creen que sigan el camino acertado (Inf. 18; 23; 24):

"Pues doy la lección, bajo contenido para descargar y ya está. Pero lo importante es que estemos todos unidos para conseguir los mismos objetivos dedicando más tiempo ellos y nosotros. La solución no es exigir menos” (Inf. 23).

Pero la mayoría sí han procurado facilitar el trabajo de sus estudiantes generando procesos interactivos (Inf. 17; 18; 21):

"Sí puedo concluir diciendo que los docentes nos han intentado transmitir emociones, principalmente de calma, sorpresa y alegría, una necesidad para que las clases fuesen motivo de esperanza y motivación” (Inf. 21).

\subsubsection{Discurso de los estudiantes sobre su corresponsabilidad}

Los estudiantes evidencian en sus narrativas ciertas experiencias que les han llevado a ser más autónomos, debidas a las transformaciones surgidas en el ámbito de la enseñanza en línea (Inf. 16; 21, 25). De modo que profundizan en su propio proceso de aprendizaje, consolidan de modo más consistente los contenidos que hay que aprender (Inf. 13, 16, 21) y son capaces de planificar y regular sus propios procesos de aprendizaje, trabajando de modo colaborativo y ejercitando, cuando ha sido necesario, la tutoría entre iguales (Inf. 19;22; 23; 25):

"Pienso que nadie estaba preparado para la situación que hemos vivido a lo largo de este año. Pero todos hemos puesto un granito de arena y nos hemos ayudado en todo lo posible. La mayoría ha dejado a un lado su egocentrismo y ha ayudado al prójimo de la manera que fuese necesario y pudiese” (Inf. 19). 
El estado de necesidad que ha generado el nuevo contexto ha dado lugar a que el alumnado desarrolle un compromiso individual y modos de hacer propios de una autonomía más madura (Inf. 3):

“Todas estas cosas hacen que a nosotros, que también nos cuesta prestar atención de esta manera, estemos más atentos y participativos” (Inf. 3).

Se observa poco sentido de corresponsabilidad en los estudiantes, incidiendo en la valoración del comportamiento del profesorado, pero no en su corresponsabilidad. Los alumnos, valoran este sentido ético del docente en consonancia con una orientación exclusivamente racional, vinculada con la divulgación del conocimiento (De La Fuente, et al., 2021). Y en este mismo sentido, los informantes que aluden a su corresponsabilidad, lo hacen en cuanto ayuda y colaboración en temas exclusivamente académicos. Lo que denota carencias importantes en la formación de la identidad profesional de los futuros maestros.

\subsubsection{Discurso de los estudiantes sobre la interacción entre profesores y alumnos}

La interacción entre estudiantes y entre estudiantes y profesores sigue orientándose, como lo ha sido el compromiso de los estudiantes, hacia el ámbito de la actividad académica (Inf. 13; 14; 16; 19), centrando la interacción en una relación fluida (Inf. 14) que queda determinada por la atención al alumnado (Inf. 16).

Ha habido, por una necesidad ineludible, un proceso de adaptación a un nuevo contexto en línea, pero que ha llevado a adaptarse a las circunstancias actuales (Inf. 13; $15 ; 19)$ :

"En cuanto al profesorado y los alumnos, se han tenido que adaptar a las circunstancias y colaborar juntos para seguir dando y recibiendo clases. Hemos tenido que interaccionar con compañeros y profesores, para poder realizar trabajo o realizar tutoría entre iguales. Ello ha facilitado la comunicación y el compromiso entre todos" (Inf. 13).

Los estudiantes han vinculado la "interacción" entre ellos y profesores como una necesidad, surgida como consecuencia de la ausencia de proximidad física, y el deseo de colaborar para salir hacia adelante, que los ha hecho ser más fuertes (Inf. 19; 24, 26):

"Las nuevas condiciones de trabajo y los problemas que genera la nueva situación han obligado a profesores y a alumnos a adaptarse y afrontar de la manera más resiliente posible esta situación. Lo que nos ha llevado a comunicarnos más entre unos y otros, como una necesidad sentida" (Inf. 24).

Se ha planteado entre los estudiantes la relación e interacción como una necesidad física y psicológica, teniendo en cuenta que muchos de ellos no se conocen, ya que hay grupos de actividad que suponen un tercio del total del grupo. Han sentido la necesidad de comunicarse ante una situación difícil, relacionándola con la finalidad de aprender, con el compromiso ético y la necesidad que tenemos las personas de comunicarnos (Inf. $9 ; 26 ; 27)$ :

“(...) me he sentido bien acogida, también por los compañeros, que a pesar de no podernos conocer como nos gustaría, desde el primer momento hemos creado una conexión muy buena y nosotros nos consideramos una piña" (Inf. 26). 


\subsubsection{Discurso de los estudiantes sobre nuevos contextos de aprendizaje}

Los estudiantes han vinculado la situación de los nuevos contextos de aprendizaje, no al desarrollo de los procesos de enseñanza-aprendizaje (preparación del profesorado), sino a las respuestas del profesorado a través de su compromiso ético con los estudiantes (Inf. 15; 6; 18):

"El profesorado y alumnado ha tenido que adaptarse a una nueva forma de conocerse, trabajar, comunicarse y evaluar, manteniendo la distancia social. Pero estoy contenta porque, dentro del cambio tan rápido que ha habido, el profesorado ha realizado un gran esfuerzo de compromiso con nosotros" (Inf. 16).

Aunque los estudiantes inciden más aún en su discurso en el acompañamiento del profesorado ante problemas generados por la situación, como estrés, soledad, problemas económicos o de motivación, o bien la inestabilidad generada por conflictos familiares, debidos a encontrarse bastante tiempo confinados (Inf. 6; 20):

"Personalmente, echo en falta ese contacto visual y cercano con los compañeros, entablar nuevas relaciones y amistades que nos enriquezcan en todos los aspectos y esta enseñanza a la que nos vemos obligados no nos ofrece eso" (Inf. 20).

Pero, sobre todo, los estudiantes aluden a las respuestas de los docentes a los problemas que genera la nueva situación de aislamiento originados por la soledad, el estrés, la tristeza o problemas económicos, entre otros (Inf. 6; 30):

"Lógicamente, esos pequeños momentos de libertad donde se nos ha permitido acudir a clase, han sido como pedacitos de cielo, porque el contacto humano nos permite olvidar problemas y situaciones que nos agobian" (Inf. 6).

"A los jóvenes se nos ha quitado la libertad propia de nuestra edad y eso ha dado lugar a que nos encontremos en una situación que va contra las características de nuestra edad, echando en falta el contacto con compañeros y encontrándonos en situaciones de estrés que produce la soledad” (In. 30).

\subsubsection{Discurso de los estudiantes sobre los problemas que genera la nueva situación}

Esta categoría se encuentra estrechamente vinculada a los nuevos contextos de aprendizaje, ya que responde a las situaciones, disfunciones y problemas que se han generado ante el nuevo formato en el que se desarrollan las enseñanzas. En primer lugar, los problemas que ha tenido el profesorado para adaptarse al uso de nuevos recursos y materiales (Inf. 15; 22; 25):

"Uno de los cambios más radicales ha estado en el profesorado, por tener que cambiar su forma de impartir las clases, como -por ejemplo- dar las lecciones mediante videoconferencia de 'meet' en vez de darlas en las clases y así sucesivamente" (15).

"El profesorado se encuentra en un momento difícil y nuevo en toda su carrera, ha tenido que enfrentarse a retos como son la incorporación de la tecnología para poder continuar enseñando a sus alumnos” (Inf. 25).

O los problemas generados por la adaptación de los estudiantes a la nueva situación académica y social (Inf. 16; 18):

"El profesorado y alumnado han tenido que adaptarse a una nueva forma de conocerse, trabajar, comunicarse y evaluar, manteniendo la distancia social. La situación de 
confinamiento que hemos vivido ha dado lugar en ocasiones al deterioro psicológico, generado por la soledad al no poder comunicarnos, la ausencia de presencia física de los compañeros o el estrés ante la incapacidad para resolver situaciones sobrevenidas” (Inf. 16).

En definitiva, el discurso de los estudiantes vincula los problemas surgidos al nuevo contexto y formato de la enseñanza, aludiendo a situaciones que ya han sido enunciadas en la categoría referida al "nuevo contexto académico", tales como: soledad, estrés, problemas económicos, o adicciones a los videojuegos. Situaciones descritas en investigaciones referenciadas (Cao et al., 2020) y evidenciadas en las narrativas de este estudio (Inf. 16; 18).

\begin{tabular}{ccccccc}
\hline & AFDL & CEP & CDE & IPA & NCA & PGNS \\
\hline AFDL & 32 & 20 & 1 & 8 & 4 & 0 \\
\hline CEP & 20 & 47 & 4 & 17 & 8 & 5 \\
\hline CDE & 1 & 4 & 8 & 3 & 0 & 2 \\
\hline IPA & 8 & 17 & 3 & 25 & 21 & 13 \\
\hline NCA & 4 & 8 & 0 & 2 & 13 & 22 \\
\hline PGNS & 0 & 5 & 0 & 2 &
\end{tabular}

Nota. AFDL: Adecuación para la formación docencia en línea; CEP: Compromiso ético del profesorado; CDE: corresponsabilidad de los estudiantes; IPA: Interacción profesores-alumnos; NCA: Nuevos contextos de aprendizaje; PGNS: Problemas que genera la nueva situación

Tabla 2. Matriz de codificación, sobre frecuencias cruzadas entre categorías temáticas, relativas al discurso de los estudiantes respecto a los nuevos contextos de aprendizaje ante el COVID-19

\section{Frecuencias cruzadas sobre contextos de enseñanza ante eI COVID-19}

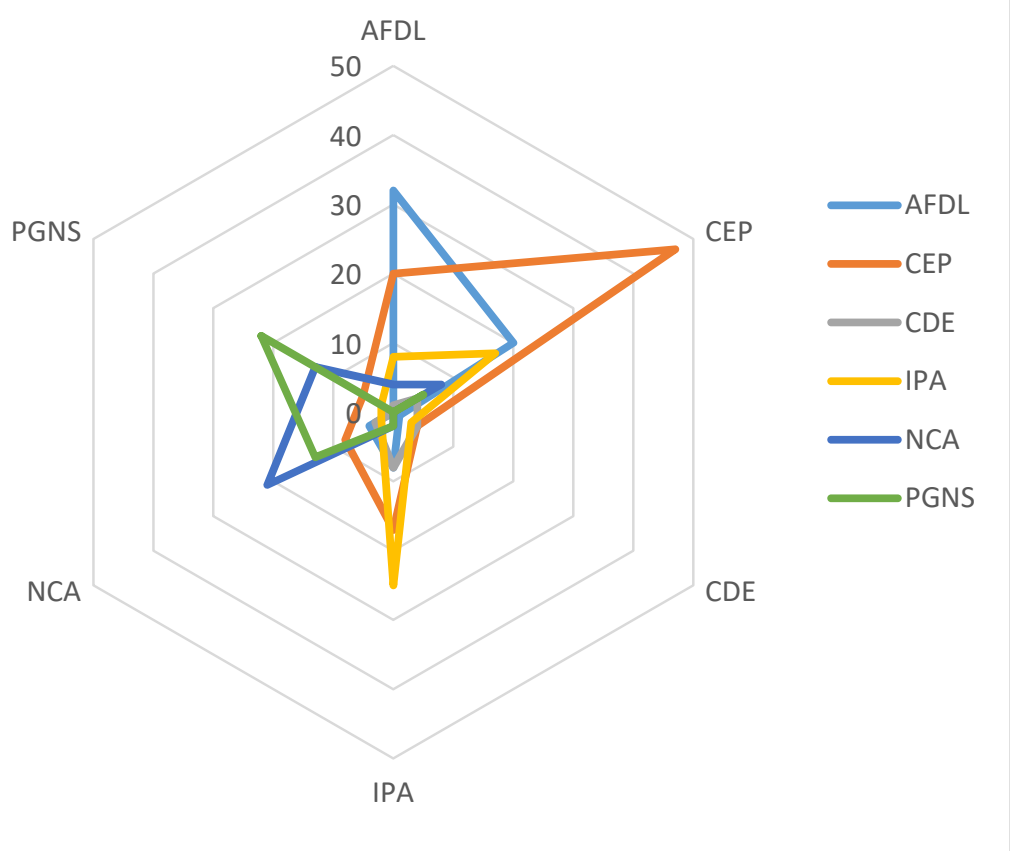

Nota. AFDL: Adecuación para la formación docencia en línea; CEP: Compromiso ético del profesorado; CDE: corresponsabilidad de los estudiantes; IPA: Interacción profesores-alumnos; NCA: Nuevos contextos de aprendizaje; PGNS: Problemas que genera la nueva situación 
Figura 2. Gráfico de redes sobre frecuencias cruzadas entre categorías temáticas del discurso de los estudiantes, respecto a los nuevos contextos de aprendizaje ante el COVID-10

\section{DISCUSIÓN Y CONCLUSIONES}

Considerando la asociación entre el marco teórico de la investigación, las narrativas autobiográficas de los estudiantes y los objetivos definidos en la investigación, se abordan los resultados más significativos de la misma.

Las narrativas de los estudiantes se han centrado en el "compromiso ético del profesorado", orientado fundamentalmente a dos categorías; la primera de ellas ordenada a la "adecuación para la formación docencia en línea", y la segunda, a "la interacción profesores y alumnos", relaciones determinadas por un marcado acento académico, concretado en la necesidad de conocer nuevos materiales y recursos, para hacer la docencia motivadora (Inf. 4; 12; 13) (Cabero y Llorente-Cejudo, 2020). La vinculación con el compromiso moral del profesorado, se centra en que sobrepasa la mera racionalidad instrumental (Buxarrais y Carreño, 2019; Martínez-Martín y CarreñoRojas, 2020), disposición cotidiana, pero que no es generalizable (Inf. 2; 5; 6, 9; 13; 18; $22 ; 31)$.

Los estudiantes vinculan el "compromiso ético del profesorado" y la mejora de la calidad de la enseñanza a la generación de procesos interactivos que faciliten un clima de comunicación favorable entre estudiantes y entre estudiantes y profesores, que posibilite el trabajo en equipo y flujos comunicativos que favorezcan un aprendizaje significativo, que demanda un logro previo de excelencia en la actividad docente, basada en la comunicación fluida, la corresponsabilidad y el conocimiento por el profesor de una epistemología práctica (Inf. 2; 6, 9; 18; 22; 31 (Alonso, 2019; MonroyFarías, 2020; Calvo y Susinos, 2010; y Martín, 2019).

La discusión de las relaciones e inferencias entre las categorías determinadas, que han dado lugar a una teoría, manifiesta ciertas inferencias, no significativas por la acumulación de su frecuencia en el discurso de los estudiantes, pero sí por la importancia que tienen en la exteriorización de la identidad ontológica de los informantes a través de su discurso.

Los informantes vinculan la solución de los problemas que se generan en los nuevos contextos de aprendizaje al compromiso ético del profesor. Sin embargo, no aluden a su corresponsabilidad. ¿Por qué sucede esto?, porque cuando se manifiestan problemas en los compañeros, surge una pulsión emocional de compasión hacia el "otro" o la "otra", que no supone el ejercicio de creencias que muevan libremente al ejercicio de juicio moral y, posteriormente, a un acto de voluntad que supone hacerse cargo del "otro", compromiso moral, que "genera una relación cuya radicalidad estriba en el vínculo que se establece entre maestro y alumno, en una situación educativa que busca la ayuda al 'otro' mediante la acogida y el compromiso" (Pérez-Ferra y Quijano-López, 2018), como se deduce de Inf. 3;19; 22;23.

Las razones por las que sucede todo ello están en la orientación filosófica y las manifestaciones culturales que orientan este tipo de discurso, sin obviar el legado que 
aportó la modernidad para superar el "pasivismo antropológico". El giro de la vertiente contemplativa a la activa no ha sido el adecuado.

La consideración de la actividad como mera efectuación, en la que la realidad teleológica de la acción está en ella misma, como constituyente de la objetividad natural, ha impedido la posibilidad de que el ciudadano sea sujeto, defina su propia identidad y no dependa de una razón arbitraria externa a la realidad personal, que elimina el desarrollo de la integralidad de cada persona. Este hecho explica y justifica el discurso de los estudiantes, en el sentido de que no trasciende lo meramente sensible y surge en aspectos más propios de su singularidad, solo cuando hay manifestaciones como tristeza, estrés etc. (Cao et al., 2020; Garris y Fleck 2020).

Más recientemente, la filosofía analítica del lenguaje, lejos de desvanecer el protagonismo que tienen los métodos científico-experimentales sobre las ciencias sociales (Bolaños, 2017) ha seguido desestructurando la identidad onto-epistemológica de las ciencias de la educación, analizando el discurso formativo desde un planteamiento fragmentario basado en la perspectiva analítico-lingüística, cuyas consecuencias ya se han podido verificar en la parcialidad de la naturaleza del discurso de los estudiantes respecto a qué es la formación y la consideración en la misma de la integridad e integralidad de la persona.

\section{BIBLIOGRAFÍA}

Alarcón-Lora, A., Munera-Cadavias, L. y Montes-Miranda, A.J. (2017). La Teoría Fundamentada en el marco de la investigación científica. Saber ciencia y libertad, 12(1), 236-245. https://doi.org/10.18041/2382-3240/saber.2017v12n1.1475

Alonso, P. (2019). El perfil del buen docente universitario desde una perspectiva del alumnado. Educação e Pesquisa, 45, 1-22. https://dx.doi.org/10.1590/s1678-4634201945196029

Álvarez-Flores, E. y Núñez, P. (2019). Efectividad del uso de herramientas digitales en el desarrollo académico de estudiantes universitarios. En S. Amavizca-Montaño (Ed.), Alfabetización informacional para la gestión del conocimiento en la Universidad (pp. 4974). Universidad Estatal de Sonora.

Apramian, T., Cristancho, S., Watling, Ch. y Lingard, L. (2017). (Re)Grounding grounded theory: a close reading of theory in four schools. Qualitative Research, 17(4) 359-376. https://doi.org/10.1177/1468794116672914

Arias-Sanabria, C.J., Carreño-Sabogal, G.A. y Mariño-Díaz, L.A. (2016). Actitiud filosófica como herramienta para pensar. Universitas Philosóphica, 33(66), 237-261. https://doi:10.11144/Javeriana.uph33-66.afhp

Aristovnik, A., Keržič, D., Ravšelj, D., Tomaževič, N. y Umek, L. (2020). Impacts of the COVID-19 pandemic on life of higher education students: A global perspectivey. Sustainability, 12(20), 1-34. https://doi.org/10.3390/su12208438

Belçaguy, M.N., Cimas, M. y Cryan, G. (2020). Usos y abusos de las TIC en estudiantes secundarios y universitarios [Ponencia]. VII Congreso Internacional de Investigación y Práctica Profesional en Psicología XXII, Buenos Aires. Argentina. Recuperado de: https://www.aacademica.org/000-015/11.pdf?view

Bolaños, R.F. (2017). El problema del conocimiento y la educación desde las tendencias filosóficas analíticas, Sophia 22, 32-52. https://doi.org/10.17163/soph.n22 .2017.01

Bossolasco, M.L., Chiecher, A.C. y Dos Santos, D.A. (2020). Perfiles de acceso y apropiación de TIC en ingresantes universitarios. Estudio comparativo en dos universidades públicas argentinas. Pixel-bit. Revista de Medios y Educación, 57, 151-172. https://doi:10.12795/pixelbit.2020.i57.06 
Brito-Lara, M. y Herrera-Rendón, E. (2021). Trabajo vía remota en tiempo de pandemia por COVID-19: condiciones y desempeños docentes en la ENSOG. En P. Chagoyán, A. Patrón, E. Herrera, M.I. Brito, C.M, Torres y J.H. Cornejo. (Eds.), Transcomplejidad y hermenéutica. Una mirada en la investigación De la formación docente (pp. 5-28). Servicio de Publicaciones de la ENSOG.

Buxarrais, M.R. y Carreño, P. (2019). Identidad moral y profesional desde una pedagogía de la proximidad. En C. Romero y T. Mateos (Eds.), Redescubrir lo educativo: nuevas miradas. Estudios en homenaje a Luis Núñez Cubero (pp. 75-86). Octaedro. https://octaedro.com/wp-content/uploads/2019/02/09072.pdf

Cabero, J. y Llorente-Cejudo, C. (2020). Covid-19: transformación radical de la digitalización en las instituciones universitarias. Campus virtuales, 9(2), 25-34. Recuperado de: http://www.uajournals.com/ojs/index.php/campusvirtuales/article/view/713/410

Calvo, A. y Susinos, T. (2010) Prácticas de investigación que escuchan la voz del alumnado: mejorar la Universidad indagando la experiencia. Profesorado. Revista de currículum y formación de profesorado, 14(3), 75-88. Recuperado de: https://www.redalyc.org/comocitar.oa? id=567157 02006

Cao, W., Fang, Z., Hou, G., Han, M., Xu, X., Dong, J. y Zheng, J. (2020). The psychological impact of the covid-19 epidemic on college students in China. Psychiatry Research, 287, 112934. http://dx.doi.org/10.1016/j.psychres.2020.112934

Coeckelbergh, M. (2020). The Postdigital in Pandemic Times: a Comment on the Covid-19 Crisis and its Political Epistemologies. Postdigital Science and Education, 2, 547-550. https://doi:10.1007/s42438-020-00119-2

Consejería de Educación, Cultura y Deporte (2015). Orden de 17 de marzo de 2015, por la que se desarrolla el currículo correspondiente a la Educación Primaria en Andalucía (BOJA de 27 de marzo). https://www.juntadeandalucia.es/boja/2015/60/1

Day, Ch, (2006). Pasión por la enseñanza. La identidad personal y profesional del docente y sus valores. Narcea.

De Dios, T. (2018). Responsabilidad de la persona y sostenibilidad de las organizaciones. Servicio de Publicaciones de la Universidad Francisco de Vitoria.

De La Calle-Maldonado, C., Miró-López, S., De Dios-Alija, S. y De La Rosa-Ruiz, D. (2021). Adaptación de la asignatura de Responsabilidad Social del grado de Periodismo a la docencia en línea, en tiempos de COVID-19. Revista de educación a distancia, 65(21), 126. http://dx.doi.org/10.6018/red.449841

De La Fuente, J., Kauffman, D.F., Dempsey, M. y Kauffman, Y. (2021). Analysis and psychoeducational implications of the behavior factor during the COVID19 emergency. Front. Psychol, 12:613881. https://doi.org/10.3389/fpsyg.2021.613881

Fernández-García, C.M., Rodríguez-Álvarez, N. y Viñuelas-Hernández, P. (2021). La percepción de los estudiantes universitarios acerca de la eficacia docente. Efectos sobre el compromiso de los estudiantes. Revista de psicodidáctica, 26(1), 62-69. https://doi.org/10.1016/j.psicod.2020.11.005

Fernández-Regueira, U., Gewerc, A. y Llamas-Nistal, M (2020). El profesorado universitario de Galicia y la enseñanza remota de emergencia: condiciones y contradicciones. Campus virtuales, 9(2), 9-24. Recuperado de:

http://www.uajournals.com/ojs/index.php/campusvirtuales/article/view/731

García-Areitio, L. (2021). COVID-19 y educación a distancia digital: preconfinamiento, confinamiento y posconfinamiento. Revista iberoamericana de educación a distancia, 24(1), 8-25. https://doi.org/10.5944/ried.24.1.28080

García-Sanz, M.P., Gomáriz-Vicente, M.Á., Hernández-Prados, M.Á. y Parra-Martínez, J. (2010). La comunicación entre la familia y el centro educativo, desde la percepción de los padres y madres de los alumnos. Educatio siglo XXI, 28(1), 157-187. Recuperado de: https://revistas.um.es/educatio/article/view/109771 
Gares, Sh., L., Kariuki, J.K. y Rempel, B.P., (2020). Community Matters: Student-Instructor Relationships Foster Student Motivation and Engagement in an Emergency Remote Teaching Environment. Journal of Chemical Education, $97(9), \quad 3.332-3.335$. https://doi.org/10.1021/acs.jchemed.0c00635

Garris, C.P. y Fleck, B. (2020). Student Evaluations os Transitioned-Online Courses During the COVID-19 Pandemic. Scholarship of Teaching and Learning in Psycholoy. https://doi. org/10.1037/stl0000229

Garrote-Rojas, D. Jiménez-Fernández, S. y Martínez-Heredia, N. (2019). El Trabajo Cooperativo como Herramienta Formativa en los Estudiantes Universitarios. Revista iberoamericana sobre calidad, eficacia y cambio educativo, 17(3), 41-58. Recuperado de: https://dialnet.unirioja.es/servlet/articulo?codigo $=6999834$

González-García., J. (2009). Inferencias en grupos infantiles de lectura”. Revista electrónica de investigación educativa, 11(1), 1-23. Recuperado de: http://redie.uabc.mx/redie/article/view/221

Herrera-Pastor, D. (2016). Aportaciones metodológicas a la investigación biográfica, relacionadas con el procesamiento y la organización de los datos. Cuestiones pedagógicas, 25, 145-160. http://dx.doi.org/10.12795/CP.2016.i25.11

Hernández-Flores, H. y Carrera-García, G. (2019). Propuesta de dos metodologías para el análisis del discurso organizacional: caso gubernamental y corporativo. Organicom, 16(30), 82-92. Recuperado de: https://core.ac.uk/download/pdf/268368093.pdf

Marciniak, R. (2015). La educación superior virtual en Polonia: Condiciones de su organización, funcionamiento y evaluación. Memorias del encuentro internacional de educación a distancia, 4, 1-13. Recuperado de:

https://www.semanticscholar.org/paper/La-educaci\%C3\%B3n-superior-virtual-en-Polonia\%3Ade-su-y-Marciniak/f792586f38b9986b9749c4bfbfd1f6c4ca51a7a8\#paper-header

Martín, P. (2019). El perfil del buen docente universitario desde una perspectiva del alumnado. Educação e Pesquisa, 45, 1-33. https://dx.doi.org/10.1590/s1678-4634201945196029

Martínez-Martín, M. y Carreño-Rojas, P. (2020). El compromiso ético del profesorado universitario en la formación de docentes. Profesorado. Revista de currículum y formación del profesorado, 24(2), 8-26. https://doi.org/10.30827/profesorado.v24i2.15150

Monroy-Farías, M. (2020). Creencias de estudiantes universitarios sobre el trabajo profesional docente. Revista de psicología electrónica Iztacala, 23(1), 128-146. file:///C:/Users/UJA/Documents/CONGRESOS\%202021/ICESD/ARTICULO/BIBLIOGRA F\%C3\%8DA/epi201g.pdf

Ortega-Ruiz, P. (2004). La educación moral como pedagogía de la alteridad. Revista española de pedagogía, año LXII, 227, 5-30. file:///C:/Users/UJA/Documents/ CONGRESOS\%202021/ICESD/ARTICULO/BIBLIOGRAF\%C3\%8DA/Dialnet-

LaEducacionMoral ComoPedagogia DeLa Alteri dad-866846.pdf

Pawluch, D. (2005). Qualitative analysis, sociology. En K. Leonard (Ed.), Encyclopedia of social measurement (pp. 231-236). Elsevier.

Pérez-Ferra, M. y Quijano-Lopez, R (2018). Análisis del discurso de los estudiantes de Magisterio sobre la contribución del practicum al desarrollo de su identidad profesional docente. Educatio siglo XXI, 36(2), 331-352. http://dx.doi.org/10.6018/j/333091

Pérez-Ferra, M., Quijano-López, R. y García-Martínez, I. (2019). Desarrollo de la competencia comunicativa en estudiantes de Bachillerato. Revista internacional de educación y aprendizaje, 7(1), 1-9. Recuperado de:

https://www.researchgate.net/publication/331043989

Pianta, R.C. y Hamre, B.K. (2009). Conceptualization, Measurement, and Improvement of Classroom Processes: Standardized Observation Can Leverage Capacity Educational Researcher, 38(2), 109-119. https://doi.org/10.3102/0013189X09332374 
Rivas, I. (2014). Nuevas identidades en la formación del profesorado: la voz del alumnado. International Journal of Developmental and Educational Psychology, 1 (17), 487-494. https://doi.org/10.17060/ijodaep. 2014.n1.v7.819

Romero, R., Riquelme, I. y Halal, C. (2019). Barreras en la percepción del maestro sobre el uso de la tecnología para la evaluación en la educación superior. Digital Education Review, 35, 170-185. https://doi.org/10.1344/der.2019.35.170-185

Shakouri, N. (2014). Qualitative Research: Incredulity toward Metanarrativeness. Journal of Education and Human Development, 3(2), 671-680. Recuperado de: http://jehdnet.com/journals/jehd/Vol_3_No_2_June_2014/40.pdf

Strauss, A. y Corbin, J. (2016). Bases de la investigación cualitativa. Técnicas y procedimientos para desarrollar la Teoría Fundamentada. Universidad de Antioquía.

Suárez-Monzón, N. (2020). Formación docente universitaria y crisis sanitaria COVID-19. CienciAmérica, 9(2), 109-114. http://dx.doi.org/ 10.33210/ca.v9i2

Tejedor, S., Cervi, L., Tusa, F. y Parola, A. (2020). Educación en tiempos de pandemia: reflexiones de alumnos y profesores sobre la enseñanza virtual universitaria en España, Italia y Ecuador. Revista latinoamericana de comunicación social, 78, 1-21. https://doi.org/10.4185/RLCS-2020-1466

Universidad de Jaén (2020). Resolución del Rectorado de la Universidad de Jaén, de 9 de noviembre de 2020, por el que se establecen las medidas a adoptar en cumplimiento del Decreto 9/2020 del Presidente de la Junta de Andalucía. https://www.ujaen.es/gobierno/viccom/sites/gobierno_viccom/files/uploads/node_book/202 0-11/Resoluci\%C3\%B3n\%20rectoral\%2009-11-2020.pdf 